\title{
La migration des particules fines comme approche d'explication du mécanisme de l'effondrement des sols
}

T. AYADAT

Maître de conférence à l'université de M'Sila

\section{B. BELOUAHRI}

Magister en Génie civil Enseignant à I'université de M'Sila

\section{R. AIIT AMMAR}

DEA en Génie civil, enseignant à l'université de Tizi Ouzou
L'étude des variations de volume est un problème majeur de la mécanique des sols. A cet effet, de nombreux chercheurs ont apporté leurs contributions à des recherches concernant l'effondrement des sols et en essayant d'en expliquer les mécanismes.

Dans le présent article, on étudie le comportement d'échantillons affaissables soumis à différentes charges hydrauliques de saturation, dans le but d'étudier et de clarifier la liaison entre la migration des particules fines d'un horizon à un autre, à travers la matrice d'un sol, et son effondrement.

Les résultats obtenus donnent des justifications et des preuves concrètes que la " suffusion » est l'un des mécanismes du (c collapse » (effondrement). On propose aussi une méthode de prévision des sols effondrables, basée sur la stabilité et l'instabilité de ces matériaux.

\section{Migration of fine size particles as a collapse mechanism of soils}

Volume changes is an important problem in soil mechanics. Several researchers have contributed in the development of researchs which concern the mechanisms of collapse of soils. In the present paper, the behaviour of collapsing samples, saturated under different pressure heads, was studied, in order to find out the relationship between the migration of fine size particles from an horizon to another, through the soil matrix, and its collapsibility.

The results obtained confirm that, the suffusion phenomenon is one of the mechanisms of collapse. A predictive method of collapsible soils, based on the stability an unstability of these materials, has been also suggested. 


\section{Introduction}

Les développements de la mécanique des sols ont jusqu'à présent, concerné principalement les sols saturés, puisque les principaux travaux ont surtout été menés dans les pays tempérés.

Cependant, la nécessité de prendre en considération le comportement des sols non saturés se fait actuellement ressentir. Initialement, cette nécessité a été mise en évidence dans les pays à climat sec ou aride, où l'évaporation est plus importante qu'une infiltration due à de faibles précipitations. Ceci entraine une variation importante des profils de teneur en eau et du degré de saturation des massifs de sol exposés sur une profondeur caractéristique qu'on appelle la zone active.

Suivant la nature du sol, ces variations peuvent entraîner des désordres dues à des phénomènes tels que l'effondrement des sols entraînant la ruine des constructions.

Le phénomène d'effondrement consiste en une densification irréversible d'un sol, initialement lâche ou peu dense, lorsqu'il est soumis à une humidification sous une contrainte totale constante. L'effondrement peut se produire aussi bien dans le cas d'un sol naturel (dépôts éoliens, dépôts alluvionnaires ou sols résiduels) que d'un sol compacté (remblais artificiels compactés dans le versant sec de l'optimum Proctor).

Pour que le phénomène d'affaissement se manifeste, il faut que le sol possède une structure apte à cet effet. D'après Barden et al. (1969), un effondrement appréciable d'un sol exige les trois conditions suivantes:

1) une structure partiellement saturée, potentiellement instable et possédant une grande porosité ;

2) une assez grande valeur des contraintes appliquées ou existantes et qui tendent à développer un état d'instabilité ;

3) l'existence d'un liant (ou d'un agent de cimentation) qui stabilise les contacts intergranulaires, qui par sa réduction ou son élimination par mouillage, produit le phénomène d'affaissement.

Tous les cas étudiés jusqu'à présent ont montré que ces sols ont une structure qui ressemble beaucoup à des nids d'abeilles, dont les grains sont liés sur place par une matière ou une force de liaison. Ces liens sont susceptibles d'une réduction ou d'une élimination en présence d'eau. Une fois le support éloigné, les grains sont capables de glisser les uns par rapport aux autres en se déplaçant vers les espaces vacants. La résistance temporaire de ces sols est pratiquement fournie par différentes matières de liaison dont les principales sont : le limon, l'argile ou des agents de cimentation.

Un grand nombre de mécanismes d'affaissement ont été imaginés, d'après les résultats obtenus sur une variété de sols :

- Knight (1961) avait supposé que la résistance des liaisons argileuses entre les gros grains se réduisait par suite d'addition d'eau à l'échantillon sous contrainte, Dès que cette résistance devient inférieure aux contraintes de cisaillement, il y a rupture de ces liaisons et l'affaissement ou l'effondrement se produit ;

- Barden et al. (1969) et Prusza, Choudry (1979) ont suggéré que l'affaissement est dû essentiellement à l'élimi- nation de la succion capillaire présente dans ces sols qui sont le plus souvent partiellement saturés ;

- dans une étude récente (Ayadat et Belouahri, 1996), il a été constaté que la granulométrie a une influence directe sur l'amplitude et le taux de l'affaissement. Il a été aussi avancé que la migration des particules fines dans le sol, d'un horizon à un autre (phénomène de suffusion), peut être l'une des causes de l'effondrement.

La présente recherche a pour but de donner des justifications et des preuves concrètes que la suffusion est l'un des mécanismes de l'effondrement. Elle est composée de trois parties :

1) essais cedométriques selon le procédé de Jennings et Knight (1975) à différentes charges hydrauliques de saturation (charges constantes), pour étudier l'influence de celles-ci sur les amplitudes d'effondrement des sols (potentiel d'affaissement CP) ;

2) essais au perméamètre à charge constante, suivant la procédure de I'US Army Corps of Engineers (1953), pour étudier l'influence des différentes charges hydrauliques de saturation sur la distribution granulométrique à des niveaux différents de l'échantillon, par rapport à celle du sol avant l'essai (état initial) ;

3) établissement, dans la mesure du possible, d'un critère de prévision des sols effondrables, en se basant sur les travaux de Kenney et Lau (1985) qui traitent de la stabilité et de l'instabilité des sols.

\section{2}

\section{Matériaux, matériels et essais}

\section{1}

\section{Matériaux de l'étude}

Les essais ont été menés sur trois sols reconstitués, composés de sable et de particules fines (inférieures à $80 \mu \mathrm{m})$ en différentes proportions, pour lesquels l'application des différents critères d'effondrement, rapportés par Ayadat et Bellili (1995), montrent que ceux-ci sont effondrables.

Le sable utilisé est un sable lavé de Oued, ses caractéristiques sont résumées comme suit :

- granulométrie comprise entre $0,08 \mathrm{~mm}$ et $2,0 \mathrm{~mm}$, dont $1,43 \%$ en poids des particules est inférieur à $0,08 \mathrm{~mm}$;

- coefficient d'uniformité : 2,14;

- coefficient de courbure : 0,95;

- module de finesse : 1,41 .

Les particules fines $(<80 \mu \mathrm{m})$ utilisées ont les caractéristiques suivantes :

- limite de liquidité : $41 \%$;

- limite de plasticité : $23,7 \%$

- densité spécifique : 2,7 ;

- pourcentage des particules inférieures à 2 microns : $21 \%$.

Les sols reconstitués ont les courbes granulométriques représentées sur la figure 1 et leurs caractéristiques géotechniques sont données dans le tableau I. 


\begin{tabular}{c|c|c|c|c}
\hline Sol & $\begin{array}{c}\text { Limite de liquidité } \\
\omega_{h}(\%)\end{array}$ & $\begin{array}{c}\text { Limite de plasticité } \\
\omega_{0}(\%)\end{array}$ & $\begin{array}{c}\text { Indice de plasticité } \\
\text { IP }\end{array}$ & Densité des grains \\
\hline S1 & 15,7 & 9 & 6,7 & 2,54 \\
\hline S2 & 21,5 & 13,4 & 8,1 & 2,58 \\
\hline S3 & 28,5 & 18 & 10,1 & 2,63 \\
\hline
\end{tabular}

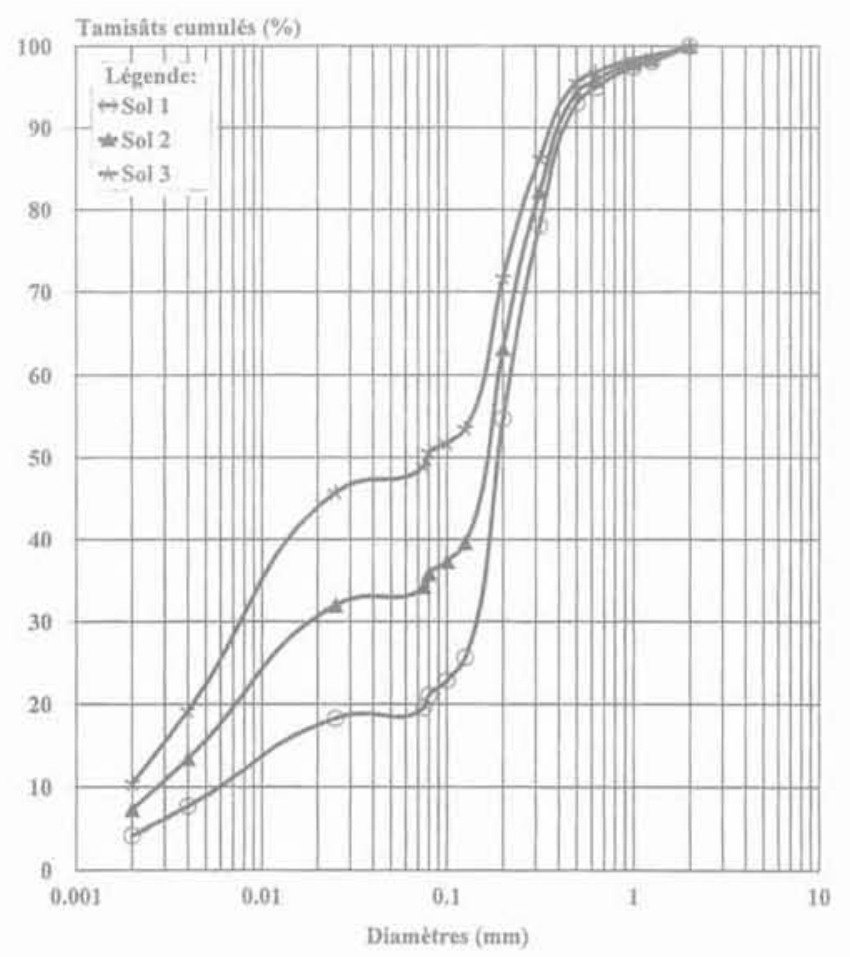

मG.1 Courbes granulométriques des sols étudiés.

Grain size distribution curves.

\section{Matériels et procédures d'essais}

\section{2,05 \\ Essais œdométriques}

Le principe consiste à reconstituer et à charger dans l'œdomètre un sol ayant une certaine teneur en eau et une certaine densité sèche. La fabrication des éprouvettes et la procédure d'exécution des essais sont décrites dans Ayadat et Belouahri (1996). La saturation des échantillons est effectuée à différentes charges hydrauliques à l'aide d'un petit réservoir dont le niveau d'eau est maintenu constant durant l'essai.

\section{2}

\section{Essais au perméamètre}

Les éprouvettes sont réalisées à partir d'un mélange de matériau de base (sable et particules fines (PF) infé-

rieures à $80 \mu \mathrm{m}$ ) bien homogénéisé. Après homogénéisation, on amène le matériau à la teneur en eau voulue par ajout de la quantité d'eau distillée nécessaire.

Après préparation d'une gâchée bien homogénéisée, celle-ci est placée dans un moule cylindrique (Fig. 2) par compactage en deux couches avec une certaine énergie (nombre de coups de mouton/couche) au moyen du dispositif de compactage décrit dans Ayadat et Belouahri (1996). Le diamètre du disque rigide de cet appareillage est d'environ $10 \mathrm{~cm}$ et la masse du mouton est de $152 \mathrm{~g}$.

Le moule cylindrique, d'un diamètre de $10 \mathrm{~cm}$ et d'une hauteur de $20 \mathrm{~cm}$, est fermé à ses deux extrémités par des couvercles; celui du bas comporte un aju-

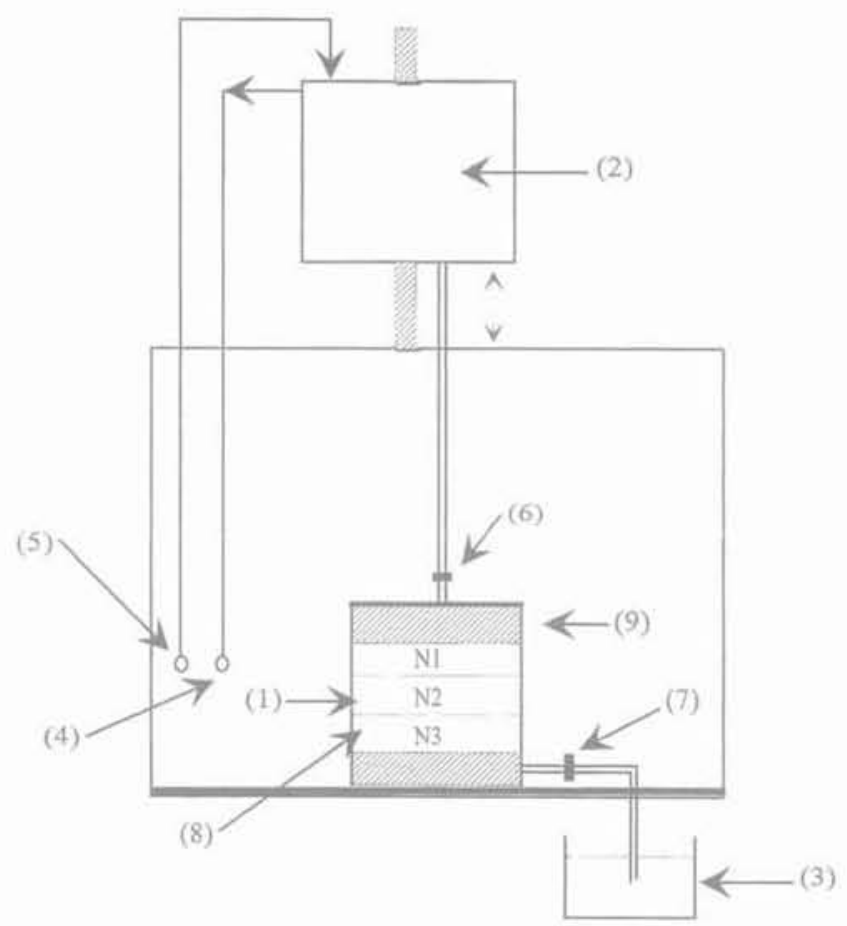

HG. 2 Perméamètres à charge constante. (1) Cellule en acier avec couvercle et découpoir (moule cylindrique)

(2) Réservoir

(3) Récipient pour recueillir l'eau drainée

(4) Vidange de trop plein

(5) Conduite pour remplissage du

réservoir

(6) Robinet $n^{\circ} 1$

(7) Robinet $n^{\circ} 2$

(8) Échantillon

(9) Pierres poreuses

Constant-head permeaters. 
tage qui sert à l'évacuation des eaux. Le couvercle supérieur est raccordé à un réservoir d'eau. Des pierres poreuses coiffent l'échantillon sur ses deux faces à l'intérieur du moule. Les essais sont effectués à charge constante où le niveau d'eau dans le réservoir est maintenu constant.

L'essai consiste à saturer et maintenir l'écoulement d'eau à travers l'échantillon pendant une durée bien déterminée. Après cette durée, l'essai est arrêté, et on laisse le sol se stabiliser pendant un certain temps. Ensuite, l'échantillon est divisé en trois couches qui sont pesées après un prélèvement représentatif pour la détermination de la teneur en eau de chacune d'entre elles.

Après tamisage humide du sol de chaque couche à travers le tamis $0,08 \mathrm{~mm}$ et séchage dans une étuve du refus, et du tamisât filtré par un filtre en papier, on procède au calcul du pourcentage PF des particules fines $(<80 \mu \mathrm{m})$ se trouvant dans chaque couche, après inondation, par la relation suivante

$$
\mathrm{PF}(\%)=\frac{\text { Masse des }(\mathrm{PF}) \text { sèches }}{\text { Masse du sol sec }}
$$

avec : Masse du sol sec $=$ Masse des $(\mathrm{PF})$ sèches + Masse du sable sec.

La masse des particules fines sèches a été calculée (à titre de comparaison) par deux méthodes :

1) masse des (PF) sèches = masse du tamisât filtré et séché :

2) masse des (PF) sèches $=\frac{\text { Masse du sol saturé }}{\left(1+w_{\uparrow}\right)}$

avec : Masse du sable $\sec =$ Masse du refus séché. $w_{1}:$ la teneur en eau de la couche $i$.

\section{3}

\section{Programme d'essais}

Les essais ont été effectués sur trois sols ayant des pourcentages de particules fines $(<80 \mu \mathrm{m})$ différents, ainsi que des énergies de compactage et des charges hydrauliques différentes. Les paramètres retenus étaient :

- Les sols d'étude :

- S1: $80 \%$ sable $+20 \%$ PF $(<80 \mu \mathrm{m})$,
$-\mathrm{S} 2: 65 \%$ sable $+35 \% \mathrm{PF}(<80 \mu \mathrm{m})$

$-\mathrm{S} 3: 50 \%$ sable $+50 \% \mathrm{PF}(<80 \mu \mathrm{m})$;

- Degrès de compactage : 10 et 30 coups :

- Teneur en eau : $4 \%$;

- Les charges hydraulique de saturation :

- essais œdométriques : 20, 60 et 100 cm,

- essais au perméamètre : 200, 240 et $280 \mathrm{~cm}$.

\section{3}

\section{Résultats et analyse}

\section{1}

\section{Essais œdométriques}

\section{Batet \\ Mise en évidence de l'effondrement des sols}

Après la préparation des sols reconstitués et la détermination de leurs propriétés physiques et mécaniques présentées dans le paragraphe précédent, on a jugé utile d'effectuer une série d'essais pour vérifier si ces sols étaient vraiment effondrables et possédaient les propriétés connues des sols effondrables naturels.

D'après les résultats des essais effectués sur les différents sols et qui sont regroupés dans le tableau II, on remarque que le potentiel d'affaissement $C P$ varie de $10,7 \%$ à $15,2 \%$ pour le sol $S 1$, de $10,9 \%$ à $15,7 \%$ pour le sol $\mathrm{S} 2$ et de $11,9 \%$ à $17,9 \%$ pour le sol S3.

Une comparaison de ces résultats avec les valeurs guides proposées par Jennings et Knight (1975) permet de classer ces différents sols dans un état de a troubles graves à différentes amplitudes d'affaissement, et ceci quelque soit le degré de compactage et le niveau de la charge hydraulique.

Pour une teneur en eau initiale donnée, le potentiel d'affaissement décroît quand on augmente l'énergie de compactage et croît quand on augmente le pourcentage d'argile contenue dans le sol. Ces résultats rejoignent ceux de Lawton (1989), Ayadat et Gherabli (1995) et Ayadat et Belouahri (1996), on peut conclure que les sols préparés artificiellement possèdent un comportement analogue à ceux rencontrés dans la nature, donc convenables pour le programme d'essais établi pour cette étude.

\begin{tabular}{c|c|c|c|c|c|c}
\hline & \multicolumn{3}{|c|}{ Potentiel daffaissement CP (\%) } \\
\hline & $\mathrm{H}=20 \mathrm{~cm}$ & $\mathrm{H}=60 \mathrm{~cm}$ & $\mathrm{H}=100 \mathrm{~cm}$ & $\mathrm{H}=20 \mathrm{~cm}$ & $\mathrm{H}=60 \mathrm{~cm}$ & $\mathrm{H}=100 \mathrm{~cm}$ \\
\hline Sol & 12,88 & 14,5 & 15,16 & 10,67 & 10,85 & 11,58 \\
\hline S1 & 14,02 & 15,05 & 15,72 & 10,93 & 11,97 & 12,74 \\
\hline S3 & 14,88 & 11,95 & 17,92 & 16,37 & 13,81 & 14 \\
\hline
\end{tabular}




\section{8}

\section{Influence de la charge hydraulique sur le potentiel d'affaissement}

Pour élucider l'influence de la charge hydraulique de saturation sur l'amplitude de l'affaissement, on a représenté sur un graphe (Fig. 3), la variation du potentiel d'affaissement CP en fonction de la hauteur de percolation d'eau. En analysant les courbes de ce graphe, nous avons pu constater que pour n'importe quel sol et n'importe quelle énergie de compactage et pour la teneur en eaux fixée à $4 \%$, l'amplitude de l'effondrement croit en augmentant la hauteur de percolation d'eau c'est-à-dire la charge hydraulique.

Ceci veut dire que la charge hydraulique de saturation a une influence directe sur l'amplitude de l'affaissement. Cela peut être expliqué par le fait que l'augmentation de la charge hydraulique fait augmenter la vitesse d'écoulement d'eau et provoque des déplacements de particules fínes dans la matrice du sol en créant des vides supplémentaires, donc des tassements additionnels causés par une diminution de volume sous une inondation et un chargement constant.

\subsection{3}

\section{Influence des particules fines sur le CP}

Pour montrer l'influence des particules fines existantes dans la composition initiale d'un sol affaissable, sur l'amplitude du « collapse », on a représenté sur la figure 4, la variation de CP en fonction du pourcentage PF des particules fines. Il est clair, d'après ce graphe, que dans les mêmes conditions, le sol contenant la plus grande quantité de particules fines exhibe le plus grand tassement (le potentiel d'affaissement le plus élevé).

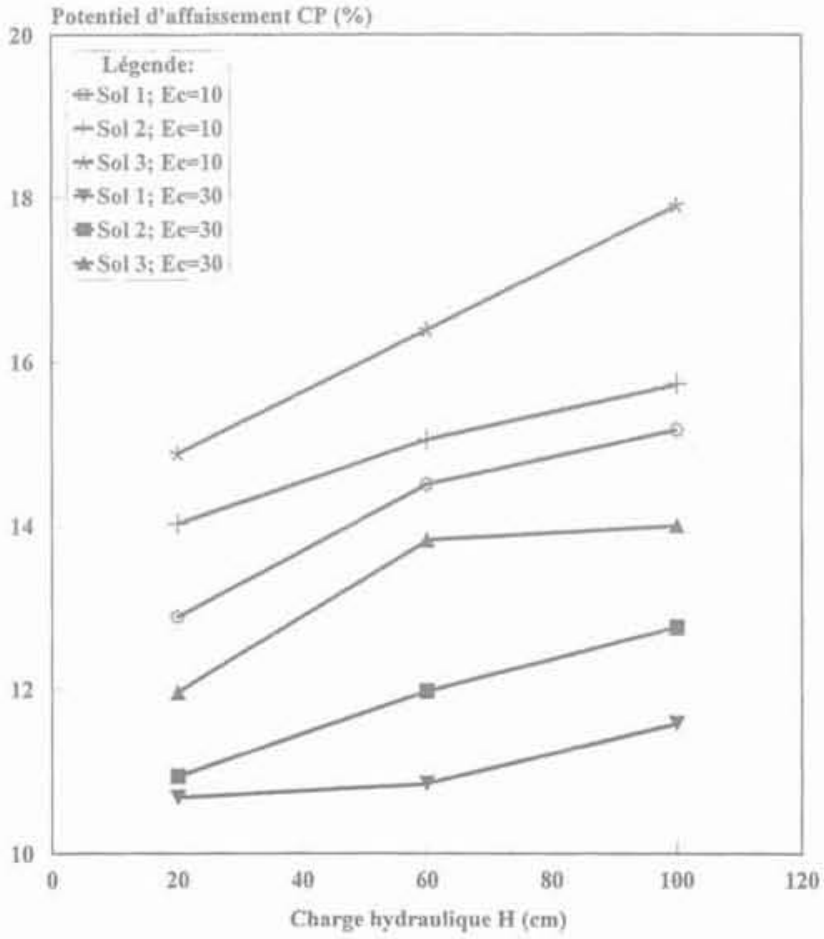

FiG.3 Variation de CP en fonction de la charge hydraulique $\mathrm{H}$.

Variation of CP against pressure heads $\mathrm{H}$.

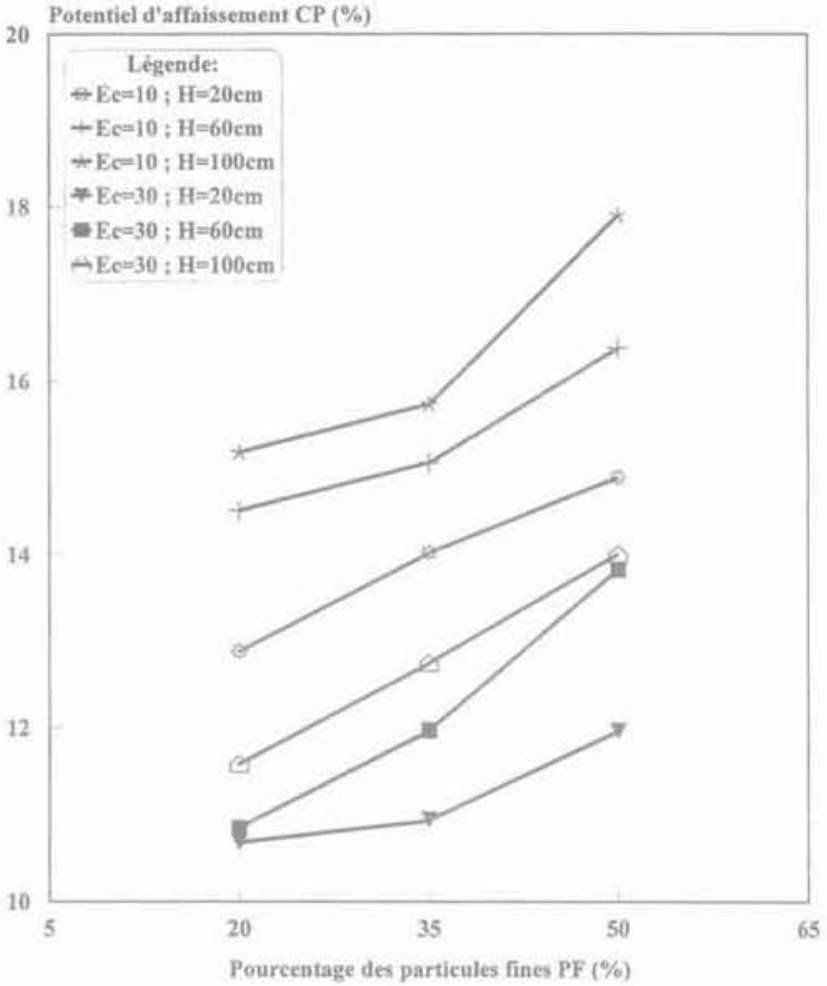

FG. 4 Variation de CP en fonction de PF.

Variation of CP against fine size particles.

Une explication de ce résultat est que la migration des particules fines dans la matrice du sol crée des vides supplémentaires qui seront de plus en plus élevés si le sol en contient une plus grande quantité.

\section{3 ât}

\section{Influence de l'énergie de compactage}

L'influence de l'énergie de compactage (10 et 30 coups) pour les différents sols est montrée dans la figure 5. D'après ce graphe, on constate que le CP décroît lorsqu'on augmente le nombre de coups. Ceci est vrai quelque soit le type de sol et la charge hydraulique de saturation.

On note aussi que l'augmentation du potentiel d'affaissement pour une énergie de compactage correspondant à 10 coups est plus grande que celle de 30 coups, pour chaque sol étudié. Le sol compacté, à une énergie de 10 coups, a une structure ouverte assez lâche avec beaucoup de vides, ce qui facilite la migration des particules fines d'un niveau à un autre à travers le sol. Réciproquement, le compactage à 30 coups rend le sol relativement dense et imperméable, la destruction des liaisons intergranulaires et le mouvement des particules fines devient relativement un peu difficile et, pourtant, les potentiels d'affaissement restent toujours élevés et correspondent aux rubriques * troubles graves $\gg$ d'après la classification de Jennings et Knight (1975).

Avant de conclure sur la liaison entre la suffusion et le phénomène de l'affaissement, il a été décidé d'effectuer des essais directs qui permettent de quantifier et de montrer clairement la migration des particules fines dans les sols effondrables. Pour cela, on a suivi 


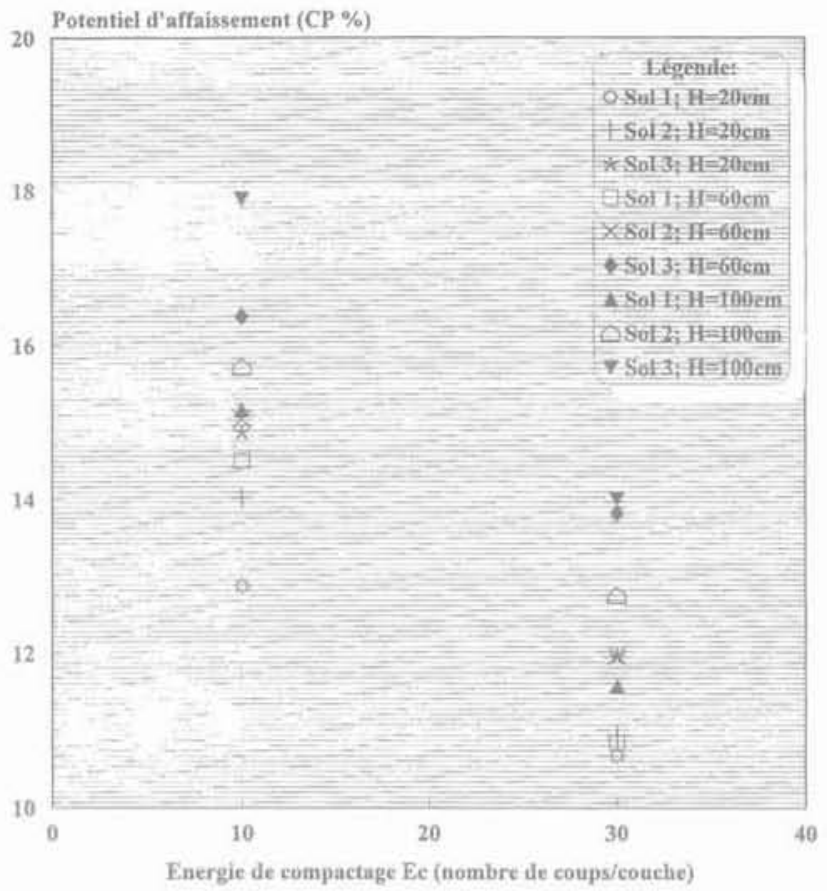

FiG.5 Influence de l'énergie de compactage Ec sur CP, pour les différents sols. Influence of the compacting energy Ec on the collapse potentiel CP, for the different soils.

l'approche expérimentale utilisée par I'US Army Corps of Engineers (1953) dans les recherches faites sur les filtres. Les essais au perméamètre décrits dans le paragraphe 2.2.2 consistent à faire une comparaison entre la granulométrie du sol à différents niveaux (après son inondation) et sa granulométrie initiale. Dans le cas présent, on s'est limité à une comparaison entre les pourcentages de PF dans les différents niveaux et leurs pourcentages dans la composition initiale du sol homogène considéré.

\section{2}

\section{Essais au perméamètre}

\section{3,29}

\section{Influence de la charge hydraulique}

L'influence de la variation de la charge hydraulique d'inondation des échantillons, pour les différentes énergies de compactages et les différents pourcentages de particules fines est montrée sur les figures 6 et 7 . On note qu'il y a une influence de la charge hydraulique sur la migration des particules fines. Lorsque la charge hydraulique augmente, on observe une diminution des $\mathrm{PF}$ dans le niveau $\mathrm{N}_{1}$, une légère augmentation dans le niveau $\mathrm{N}_{2}$ et une augmentation remarquable dans le niveau $\mathrm{N}_{3}^{2}$.

Autrement dit, chaque fois qu'on augmente le niveau de percolation d'eau (donc la vitesse d'écoulement) on remarque que la diminution des particules fines dans le niveau $\mathrm{N}_{1}$ est plus grande et que l'augmentation des PF dans les niveaux $\mathrm{N}_{2}$ et $\mathrm{N}_{3}$ est plus élevée.
Pourcentage des particules fines $(\%)$

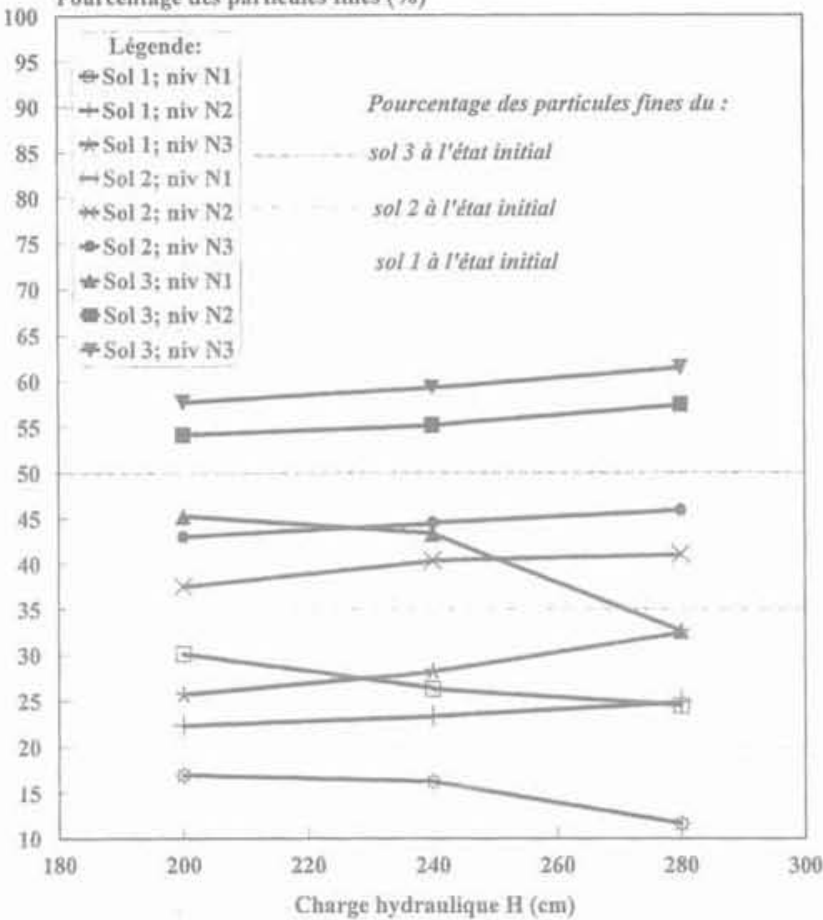

FG.6 Influence de la charge hydraulique $(\mathrm{H})$ sur la migration des particules fines, pour Ec $=10$ coups.

Influence of the pressure heads $(\mathrm{H})$ on the migration of fine size particles, for $\mathrm{Ec}=$ 10 blows.

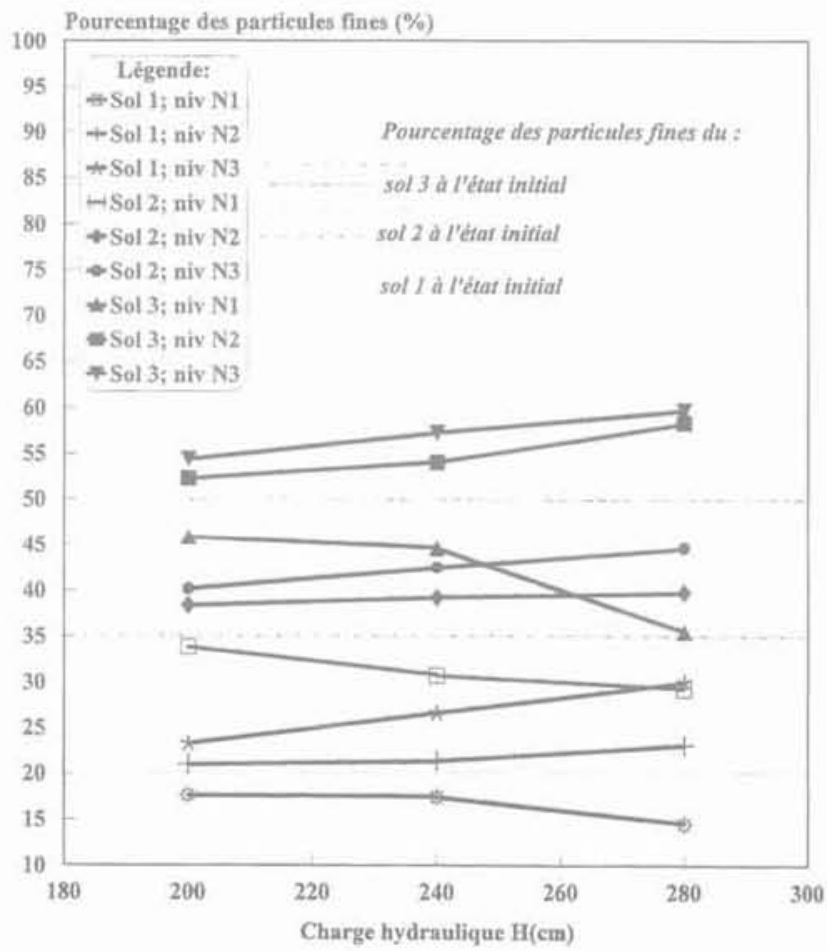

F10.7 Influence de la charge hydraulique $(\mathrm{H})$ sur la migration des particules fines, pour $\mathrm{Ec}=30$ coups.

Influence of the pressure heads $(\mathrm{H})$ on the migration of fine size particles, for $\mathrm{Ec}=30$ blows. 
Donc, juste après l'élimination des liaisons intergranulaires par contact avec l'eau, les particules fines se déplacent dans le sol d'un niveau à un autre.

\section{$3=3.29$ \\ Influence des particules fines}

On définit la différence (D) entre les pourcentages des particules fines $(<80 \mu \mathrm{m})$ contenues dans le niveau $\mathrm{N}_{1}$ et les autres niveaux de l'échantillon, après saturation, par la relation:

$$
D_{i}=P F\left(N_{i+1}\right)-P F\left(N_{1}\right)(\%)
$$

oú : $P F\left(N_{1}\right)$ : pourcentage des particules fines $(<80 \mu \mathrm{m})$ contenues dans le niveau $\mathrm{N}_{1}$ de l'échantillon après saturation :

PF $\left(N_{i, 1}\right)$ : pourcentage des particules fines $(<80 \mu \mathrm{m})$ contenues dans le niveau $\mathrm{N}_{2}$ ou dans le niveau $\mathrm{N}_{3}$ de l'échantillon après saturation.

Pour montrer l'influence de la quantité cies particules fines $(<80 \mu \mathrm{m})$ contenues dans les sols à l'état initial sur leur migration à travers la matrice de ces sols, on a représenté sur les figures 8 et 9 , la variation de $\mathrm{D}$. en fonction de la charge hydraulique $(\mathrm{H})$ pour les différents sols et les différentes énergies de compactage. D'après ces figures, on peut faire les constatations suivantes :

- les différences $D_{1}$ et $D_{y}$, représentant respectivement les différences entre les pourcentages des particules fines des niveaux $\mathrm{N}_{2}-\mathrm{N}_{1}$ et $\mathrm{N}_{3}-\mathrm{N}_{1}$, sont d'autant plus grandes ou plus importantes que la quantité de particules fines, contenue dans la granulométrie initiale du sol est plus élevée :

- la différence entre $D_{2}$ et $D_{1}$ croît avec la charge hydraulique de saturation.

Ces deux constations montrent clairement qu'il y a une dépendance entre la migration des particules fines dans un sol affaissable et sa granulométrie initiale, ainsi qu'avec la charge hydraulique de saturation.

\section{$\sqrt{3.983}$}

\section{Influence de l'énergie de compactage}

D'après les figures 6 et 7 , on remarque que l'état initial de compacité du sol a aussi une influence sur le taux de migration des particules fines, et ceci quelque soit la charge hydraulique de saturation. C'est-à-dire que les particules fines se déplacent facilement et en grande quantité lorsque le sol est à l'état lâche. Ce déplacement devient de plus en plus difficile lorsque le sol passe d'un état lâche à un état relativement compact (30 coups). Donc, on peut dire qu'un sol compact est peu ou non affaissable à cause de sa faible porosité et parce que la migration des particules fines à travers sa structure devient faible.

Les résultats obtenus dans cette recherche montrent qu'il y a une relation, d'une part, entre la charge hydraulique de saturation et le potentiel d'affaissement et, d'autre part, entre la charge hydraulique et la migration des particules fines, à travers la matrice d'un sol affaissable, d'un niveau à un autre. Donc, on peut conclure que l'affaissement des sols effondrables est lié à la migration des particules fines à travers leurs structures. C'est-à-dire que la suffusion est l'une des causes du "collapse ".

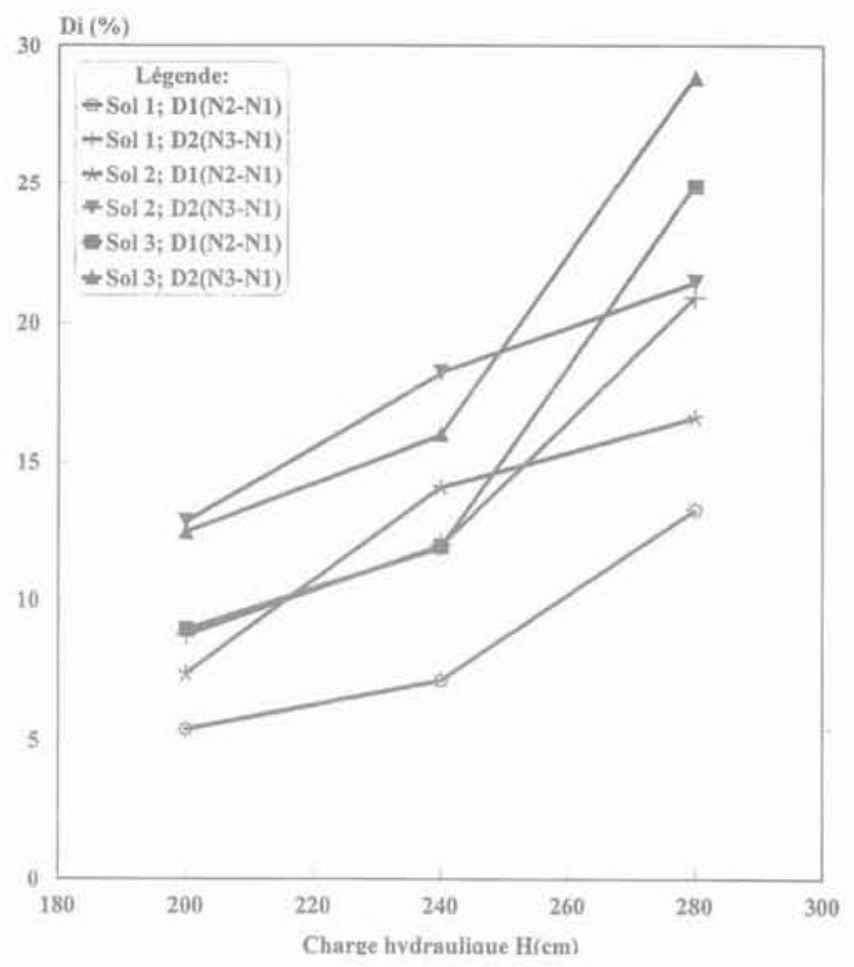

nG. 8 Variation de la différence Di en fonction de la charge hydraulique pour différents sols $(\mathrm{Ec}=10$ coups).

Variation of Di against pressure heads for the different soils $(\mathrm{Ec}=10 \mathrm{blows})$.

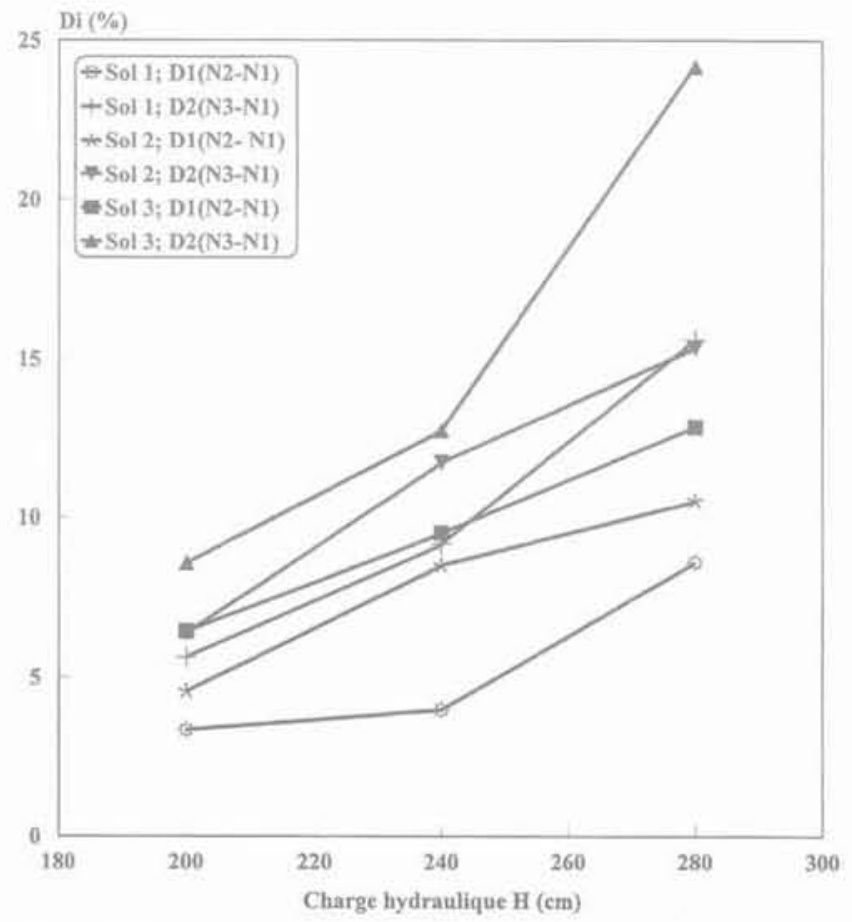

FIG 9 Variation de la différence Di en fonction de la charge hydraulique pour différents sols $(\mathrm{Ec}=30$ coups).

Variation of Di against pressure heads for the different soils ( $\mathrm{Ec}=30$ blows). 


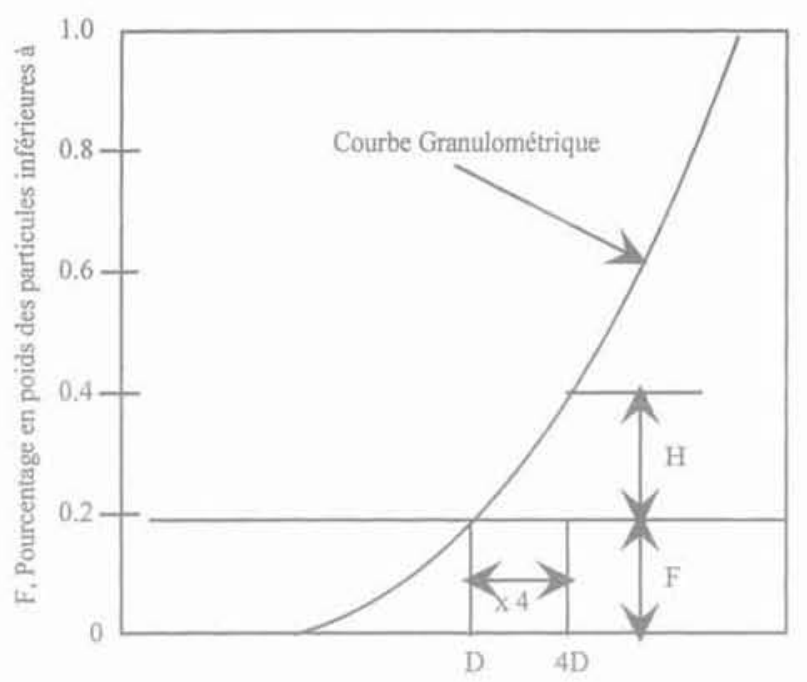

Diamétre D

(Echelle Logarithmique)

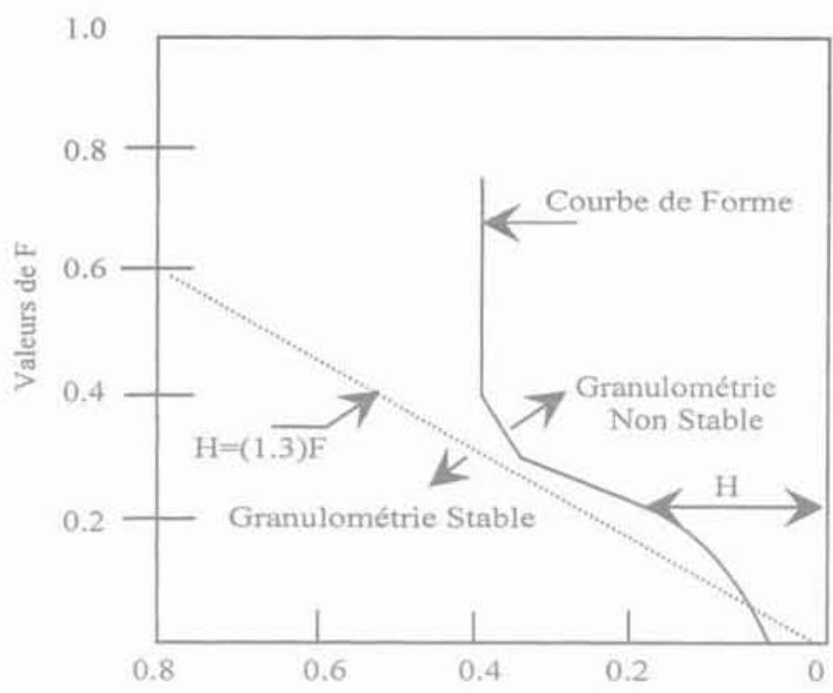

$\mathrm{H}$, Pourcentage en poids des particules de diamétre entre $\mathrm{D}$ et $4 \mathrm{D}$

FG. 10 Méthode de description de la forme de la courbe granulométrique (d'après Kenney et Lau, 1985). Method of describing the shape of grading curve (after Kenney and Lau, 1985).

\section{3}

\section{Proposition d'une méthode de prévision des sols effondrables}

Les diverses observations précédentes et celles mentionnées dans Ayadat et Belouahri (1996) ont confirmé que l'effondrement est en liaison avec le phénomène de suffusion. L'instabilité des sols effondrables, causée par le mouillage, est due à la migration des particules fines à travers leurs structures. Ce résultat nous a incité à exploiter la méthode proposée par Kenney et Lau (1985) pour l'identification des filtres stables, et instables, pour prévoir si les sols sont effondrables puisque ce phénomène est lié à l'instabilité de leurs structures sous inondation.

La méthode consiste à tracer la variable $\mathrm{F}$, dans un repère orthonormé, en fonction de $\mathrm{H}$ où :

- F: pourcentage en poids des particules dont le diamètre est inférieur à D ;

- D : diamètre quelconque des particules compris entre $\mathrm{D}_{0}$ et $\mathrm{D}_{100}$;

- H : pourcentage en poids des particules dont le diamètre est compris entre $\mathrm{D}$ et $4 \mathrm{D}$.

$$
\mathrm{H}=\mathrm{F}_{4 \mathrm{D}}-\mathrm{F}_{\mathrm{D}}
$$

La droite d'équation: $\mathrm{H}=1,3 \mathrm{~F}$, sépare les sols stables des sols instables (voir Fig. 10). Si la courbe représentative du sol se situe au-dessus ou coupe cette droite, le sol est considéré comme instable.

On a appliqué cette méthode pour un certain nombre de sols effondrables connus, et on a vérifié que ces sols ont, d'après leurs granulométries, des structures instables (Fig. 11a et b). Ce résultat peut être utilisé pour identifier ou prévoir les sols susceptibles à l'effondrement en connaissant leurs compositions granulométriques. Un sol effondrable est un sol instable, susceptible de suffusion pour qui la représentation de sa courbe granulométrique dans le diagramme de Kenney et Lau coupe la droite $\mathrm{H}=1,3 \mathrm{~F}$ (la zone des sols instables).
Il est indispensable de noter, d'après ce graphe, que le sol est d'autant plus instable (susceptible du phénomène de suffusion), qu'il contient plus de particules fines, ce qui concorde avec son potentiel d'affaissement élevé.

Autrement dit, un sol est d'autant plus susceptible au phénomène de suffusion donc à l'effondrement que sa courbe granulométrique est étalée. Ceci concorde avec les résultats de Ayadat et Belouahri (1996) qui sti-

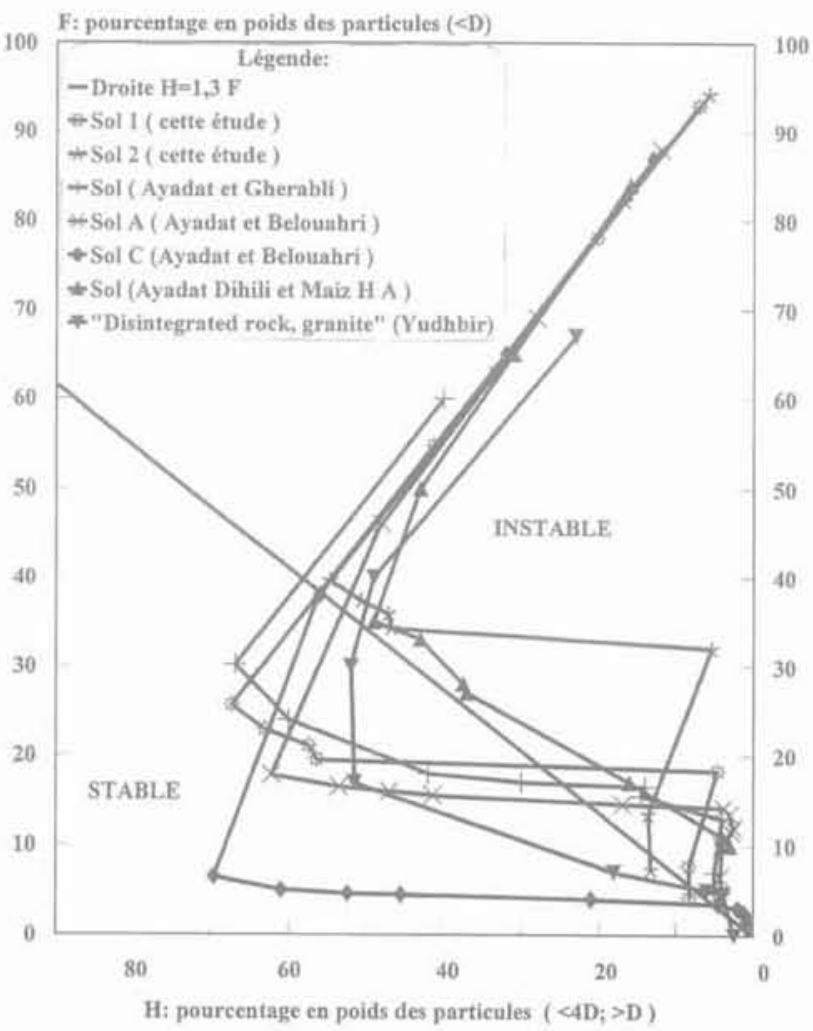

FIG. 110 Application pour quelques sols effondrables connus. Application of the method for some collapsing soils. 
F: pourcentage en poids des particules $(<D)$

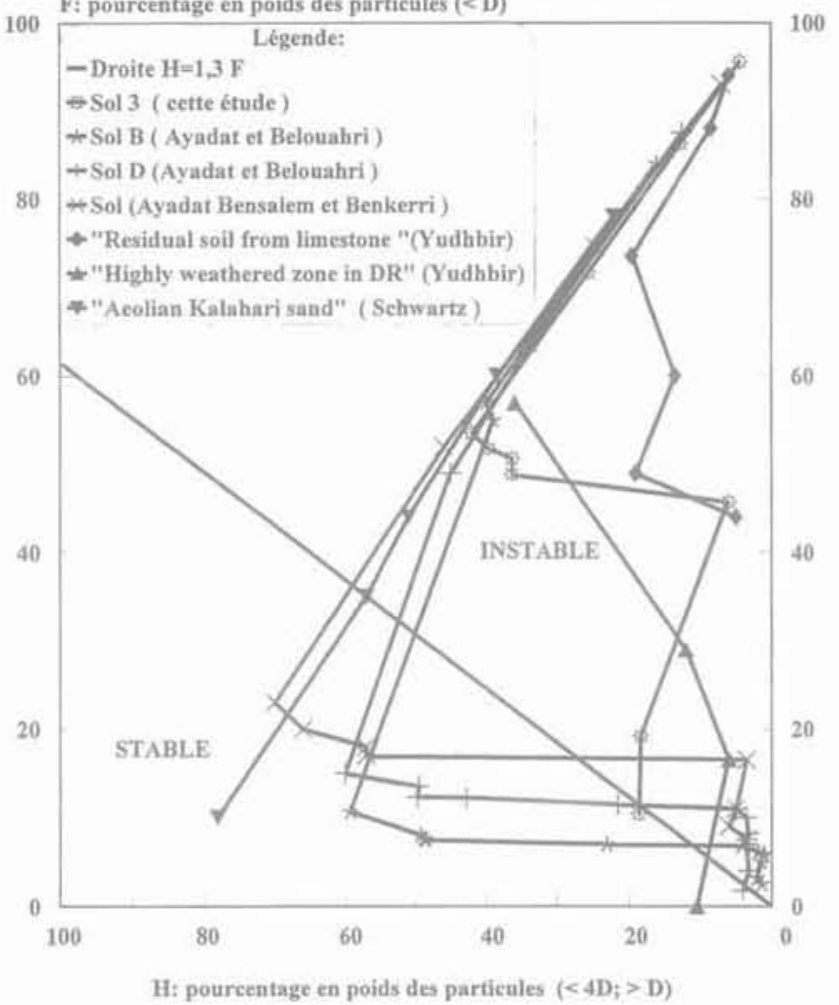

FIG. 116 Application pour quelques sols effondrables connus. Application of the method for some collapsing soils. pulent qu'un sol est d'autant plus susceptible à l'effondrement que son coefficient d'uniformité est plus élevé.

\section{4}

\section{Conclusion}

Les principales conclusions qu'on peut tirer de cette étude se résument comme suit:

1. Il y a une influence marquée de la charge hydraulique de saturation sur le comportement des sols effondrables. Le potentiel d'affaissement croit lorsque la charge de percolation augmente. Cette croissance est d'autant plus importante que les sols sont dans un état lâche et contiennent une grande quantité de particules fines.

2. Il a été montré que, pendant l'inondation des sols effondrables, les particules fines se déplacent à travers leurs structures d'un niveau à l'autre (phénomène de suffusion): Cette migration dépend généralement de la granulométrie du sol, de sa compacité initiale et de la charge hydraulique de saturation.

3. L'effondrement est lié au phénomène de suffusion. Il a été confirmé par cette recherche que l'effondrement des sols sous mouillage est causé par la migration des particules fines, après élimination de la cohésion apparente.

4. Une nouvelle méthode de prévision des sols effondrables a été proposée. Cette méthode utilisée pour identifier les filtres stables et instables, est basée seulement sur la connaissance de la granulométrie des sols à étudier.

\section{$\overline{\text { Bibliographie }}$}

Ayadat T., Bellili F. - a Sols susceptibles d'affaissement : identification, mécanismes et traitement $\mathrm{D}$. Revue AlgérieÉquipement, n²0, juillet/août 1995, p. 18-23,

Ayadat T. Bensalem A. Benkerri A. «Traitement d'un sol affaissable par la chaux ». Revue Française de Géotechnique, $\mathrm{n}^{\circ} 77,4^{*}$ trimestre 1996.

Ayadat T., Belouahri B. $-\alpha$ Influence du coefficient d'uniformité sur l'amplitude et le taux de l'affaissement des sols $n$ Revue Française de Géotechnique, $\mathrm{n}^{\circ} 76$, $3^{e}$ trimestre 1996, p. 25-35.

Ayadat T, Dhili M, Maiz Hadj Ahmed K « Traitement d'un sol effondrable par un liant hydrocarboné (bitume) y. Article remis à la Revue Française de Géotechnique, 1997.
Ayadat T., Gherabli A. - $\alpha$ Traitement des sols affaissables par du ciment CPA x. Annales de ITTBTP, $n^{\circ} 530$, janvier 1995 . p. 34-43.

Barden L... Madedar A.O., Sides G.R. "Volume change characteristics of unsaturated clay ». Journal of SMFD. ASCE, vol. 95, SM1, 1969, p. 33-49.

Jennings J.E. Knight K. - a. A guide to construction on or with materials exhibiting additional settlement due to collapse of grain-structure $v$. Proc. 6th Regional Conf. for Africa on SMFE, Durban. South Africa, 1975, p. 99-105.

Kenney P.C., Lau D. - $\alpha$ Internal stabily of granular filter x. Canadian Geotechnical Journal, vol. 22, 1985, p. 215-225.

Knight K (1961) - (reporté par Yudhbir, Collapsing, behaviour of residual soils) Proceeding of the 7 th Southeast Asian
Geotechnical Conference, Hong Kong, 1982, p. $915-930$

Lawton E.G. Fragaszy R.J., James H.H. $\alpha$ Colapse of compacted clayed sand $x$. J. of Geot. Eng. Div., ASCE, vol. 115, n9, 1989, p. 1252-1267.

Schwartz K. Yates J.R.C. Tromp B.E. - $\alpha$ In situ Compaction of Aeolian Kalahari Sand $x$. Proc of the 8th Int. Conf. of Soil Mech and Found. Eng, Moscow, USSR, 1973, p. 773-777.

U.S. Army Corps of Engineers - $\alpha$ Filter Experiments and Design Criteria n. Technical Memorandum $n^{\circ} 3-360$. U.S Army Waterways Experiment Station, Vicksburg, Miss, Apr. 1953.

Yudhbir - «Collapsing behaviour of residual soils $x$. Proceeding of the 7th Southeast Asian Geotechnical Conference, Hong Kong, 1982, p. 915-930. 